\title{
Mathematical Etudes: Evolution from Multimedia to a Book
}

N. N. Andreev, N.P. Dolbilin, S. P. Konovalov and N. M. Panyunin (Steklov Mathematical Institute, Moscow, Russia

An exceptional feature of mathematical popularisation in Russia from its very beginning has been the main role always played by leading scientists. The popularisation movement started from extracurricular clubs called "mathematical circles", which appeared in Leningrad (now Saint Petersburg) in the early 1930s. This maths club activity was accompanied by the first Russian citywide Mathematics Olympiad for high school pupils, organised in 1934 by the prominent mathematician and populariser Boris Nikolaevich Delaunay. In the post-war period, the maths circles and mathematics Olympiad movement became a mass phenomenon. Many outstanding mathematicians (including A.N. Kolmogorov, P.S. Alexandrov, I.M. Gel'fand, D.K. Faddeev and V.I. Arnold) were engaged in this activity: giving lectures, writing popular science books for children and organising Olympiads. It was understood (and this fact also became Russian tradition) that to awaken a child's interest in some occupation, it had to be taught by leading scientists.

Following these Russian traditions in popularising mathematics, the Steklov Mathematical Institute, through the initiative of its director academician V.V. Kozlov, launched in 2010 a separate Laboratory of Popularisation and Promotion of Mathematics (though preparatory work preceding its creation began in 2002). This Laboratory became a pioneer in the promotion of mathematics in Russia, setting new standards in the popularisation of mathematics and stimulating the development of this field.

During the first years of the Lab, several multimedia projects were established that are now well known in Russia. A list of these projects is presented in more detail below.

- Mathematical Etudes (ME). This is a series of more than 60 movies, made using modern 3D computer graphics, devoted to some solved and unsolved mathematical problems.

- Mechanisms by Tchebyshev. This is a set of movies and other materials on mechanisms suggested and constructed by this great Russian mathematician of the 19th century.

- Apps for mobile devices with mathematical content.

Several electronic online libraries were also organised. The first of them is the archive of the Publishing House "Mathesis", which was the first publishing house in Russia publishing the best popular science books on physics and mathematics printed in Europe and Russia from 1904 to 1925.
The second online library is the archive of the $E x$ perimental Physics and Elementary Mathematics Bulletin journal, which was published 24 times a year from 1886 to 1917. This journal was a pre-revolutionary analogue of the famous magazine Kvant, which was organised under the guidance of the eminent scientists I.M. Kikoin and A.N. Kolmogorov in 1970 and still exists to this day. The magazine Quantum, published in the US from 1989 to 2002 , consisted of selected materials from Kvant.

It is surprising (taking into account that a century has passed since then) how many interesting materials on mathematics and the other sciences can still be found in these books and journals. These materials remain useful for the popularisation of both science and education. (Unfortunately, the last two projects are only available in Russian.)

\section{"Mathematical Essence"}

For the first 13 years, the team of the Laboratory showed up mostly on the internet, even though they also delivered hundreds of lectures for schools and university students, and teachers and educators in various regions of Russia. It should be emphasised that these lectures were all based on the multimedia content created in the Laboratory.

However, in 2015, the Laboratory found it necessary to prepare a book called Mathematical Essence. Its publication was supported by the Scientific Council of Steklov Mathematical Institute. The Russian title of the book is not easy to translate adequately into English, giving several possible alternative English versions: "Mathematical Component" "Mathematical Feature" or "Mathematical Constituent".

The fact that mathematics is the language and an important tool of science and technology is well known to any reader. The experienced reader will know that mathematics plays a great role in physics, in the implementation of space flight, in the taming of atomic energy and in the computer world. The importance of mathematics in other fields such as medicine or linguistics is less known to the general public.

But even the reader who has heard about the significant mathematical "component" in various spheres of human activity is often not aware of the degree of dependence of these sciences on mathematics. The main reason for such ignorance is the complexity of mathematical tools designed particularly for a given application. Verbally recognising the role of mathematics, people usually do not ponder the mathematical "filling" of the objects and phenomena surrounding us. Sometimes they simply do not notice it. 
The main purpose of the book Mathematical Essence is precisely to bring to the surface the mathematical "essence" of some of the greatest achievements of our civilization, as well as to manifest the mathematical "content" inside usual, everyday things.

There is one more problem in modern day life that should be taken into account by popularisers of mathematics: information overload, resulting in an almost universal unwillingness to learn anything that is not related to a daily necessity. From this point of view, presentation of mathematics as a necessary and essential part of world knowledge may produce a "personal" interest in the potential reader for studying mathematics.

The authors of the book are well known as actively working mathematicians. It is very important that the reader obtains all the scientific information from "the table" of leading scientists. A popular descriptive style of presentation (with minimal use of formulas) is specially designed for a wide range of readers.

It was clear to the editors that the publication of such a book was absolutely necessary and timely, noting that the range of readers prepared to read popular books on mathematics has been rapidly decreasing over recent decades and we should respond in some way to this sad tendency. One of the purposes of the book is to show that mathematics is not an isolated science but an essential (although sometimes hidden) part of many important phenomena and objects of the world. The illustrations presented and the Russian website http://book.etudes.ru give an idea of the topics collected in the book.

Why did we decide to return to the classical format of a book after working for over 10 years on the creation of multimedia presentations of mathematical topics? The main reason is that all the Laboratory materials were designed for a thinking person and it is much more convenient to think while reading a book rather than clinging to the monitor screen.

There is another psychological advantage of a book compared to multimedia and interactive sites: a reader, looking through the contents of the book, gets an immediate idea of the book as a whole.

And finally, to illustrate one more reason for the evolution from multimedia to a book, one could say that the difference between a website and a book is the same as the difference between a young and a vintage wine. The contents of a book (and the authors themselves) need to mature before a decent publication.

In Russia, "Mathematical Essence" has become very popular and has won a prestigious prize for popular scientific literature. We hope that the potential translation of the book into other languages will expand the circle of its readers.

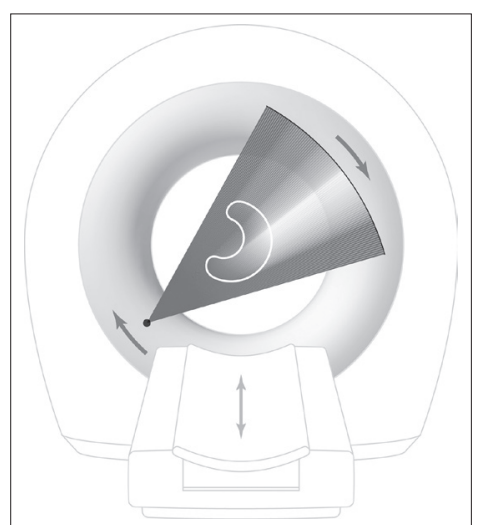

Computer tomography (by A. G. Sergeev).

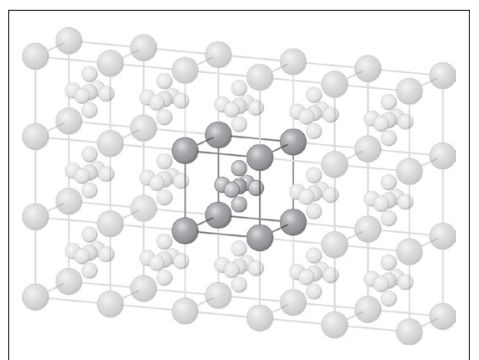

Geometric crystallography (by N.P. Dolbilin).

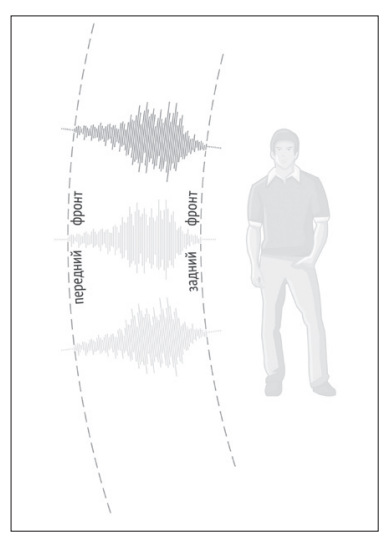

Sound wave propagation (by M.V. Feigin).

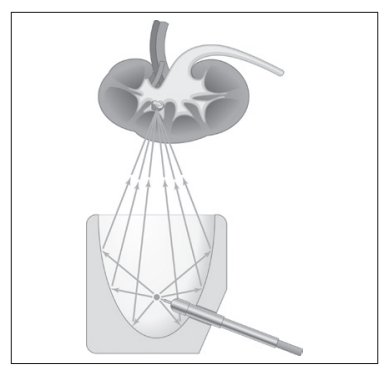

Kidney stone treatment (optical properties of an ellipse).

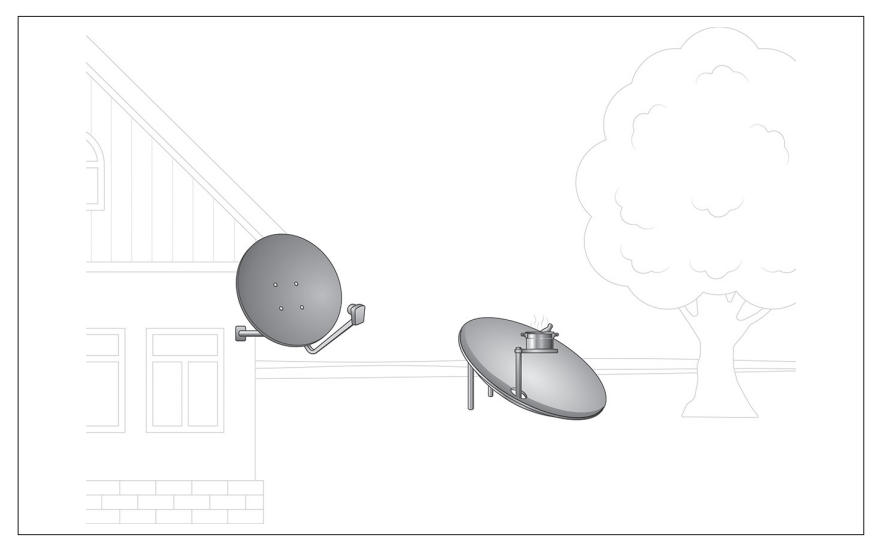

Parabolic antenna (optical properties of a parabola).

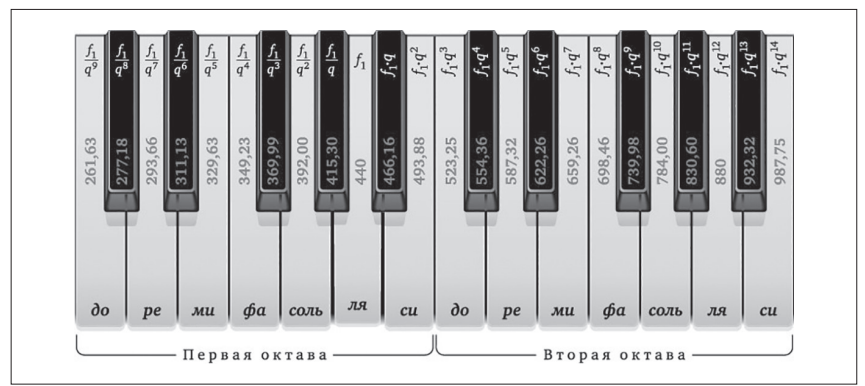

The musical scale (geometric progression).

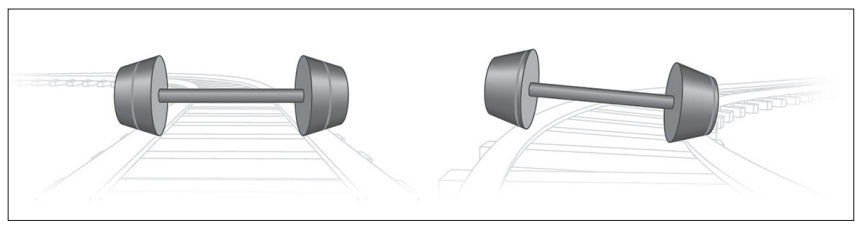

Railway wheel sets (properties of a cone). 


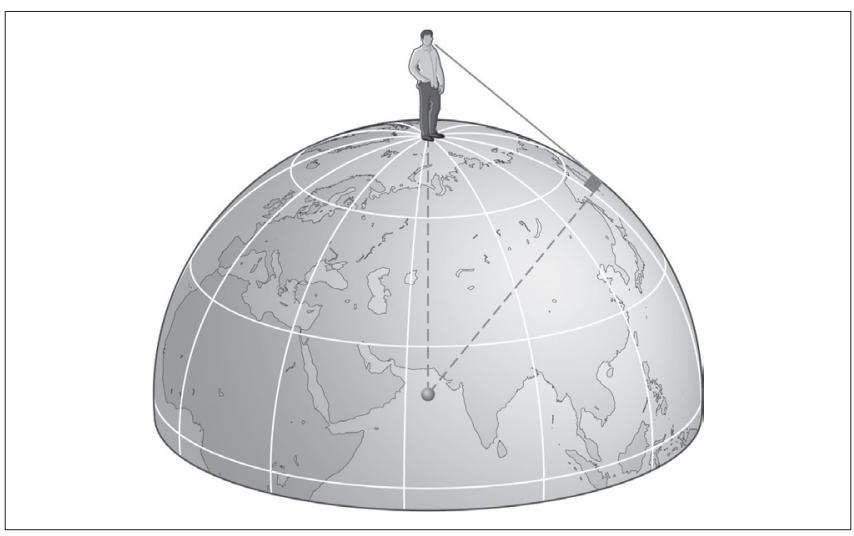

Distance to the horizon (the Pythagorean theorem).

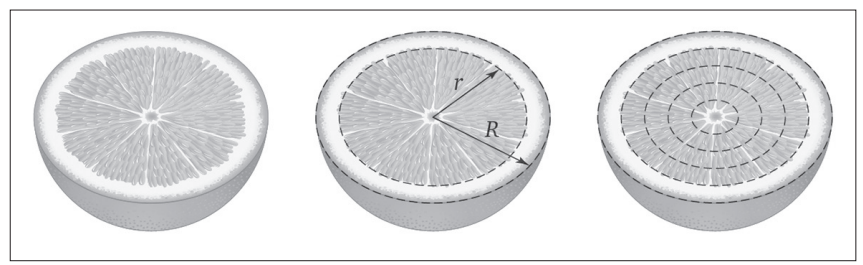

Volume of oranges (properties of the volume of a ball).

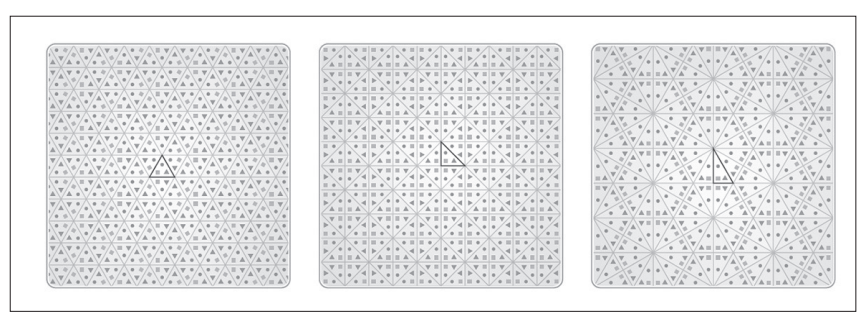

Kaleidoscope (reflection groups).

\section{"Mathematical Etudes"}

More than 60 movies on different topics in pure and applied mathematics have been created throughout 13 years of the main Laboratory project "Mathematical Etudes" (http://etudes.ru). Some of the movies are based on purely mathematical ideas; the others deal with its applications to technology and engineering. There are also movies that describe the historical context of a discovery and present the scientists and engineers who made crucial contributions. Each movie is accompanied by a short popular description of the problem and appropriate references and links. The main goal is to attract viewers to mathematics, to show its intrinsic beauty and present ideas for a deeper understanding of the world.

This project differs from the other, more traditional forms of popularisation by the intensive use of modern tools in the presentation. The main tool is the use of 3D computer graphics, which has been chosen not only because it makes the mathematical ideas easier to understand but also because it is much more attractive for today's young people.

Some highly experienced computer professionals have gathered together for this project: the 3D graphics were produced by Mikhail Kalinichenko, and Roman Koksharov created the 2D graphics, as well as designing and programming the websites (not only for this project but also for our other projects).
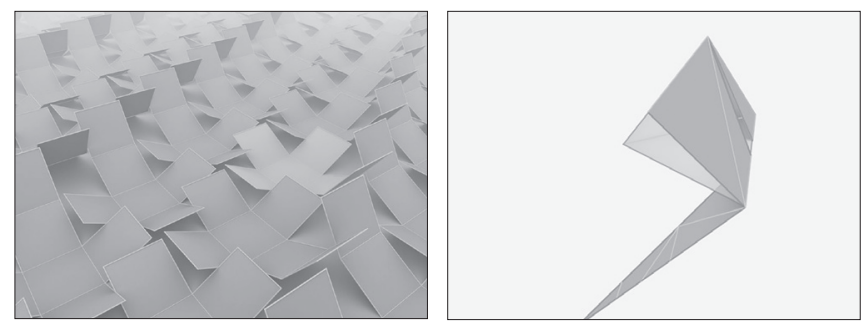

Cubist parquet (nets of the cube). And this is a net?! (the theory of nets of polyhedra).

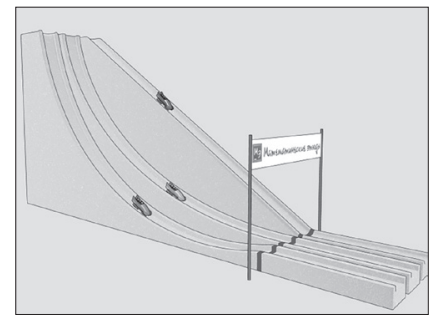

Cycloid (the brahistohrone problem).

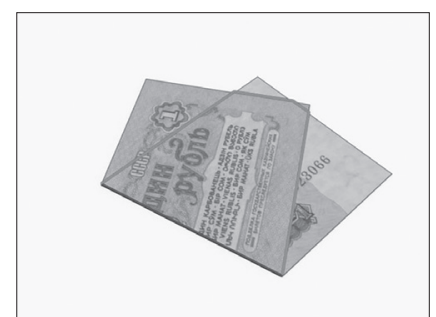

The folded rouble (V.I. Arnold's problem).

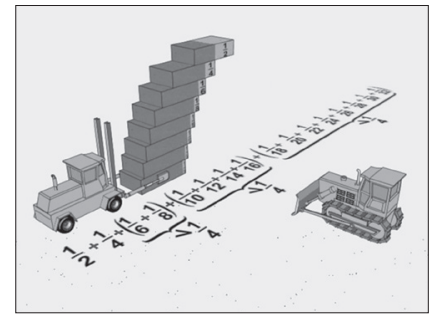

A ladder to infinity (the divergence of the harmonic series).

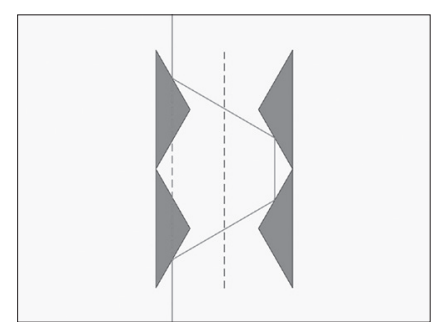

The invisible (construction of invisible bodies).

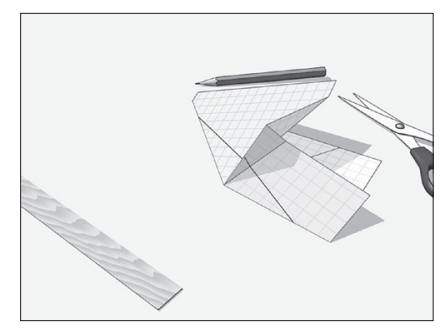

With a single cut (cutting out an arbitrary polygon with one incision).

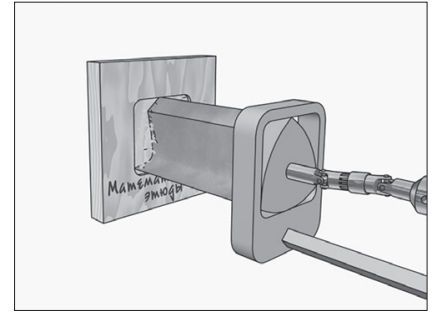

Drilling of square holes (figures of constant width).

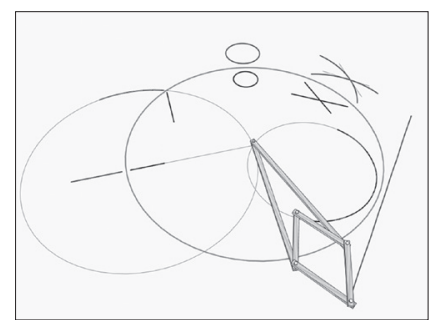

Lipkin inversor (how to draw a straight line with linkage).

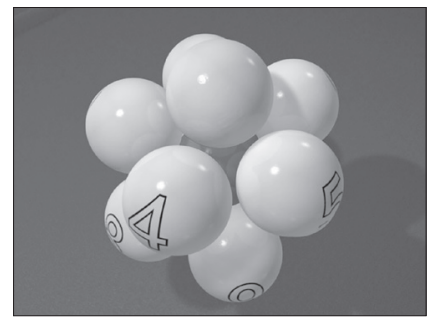

Contact numbers (the theory of coding).

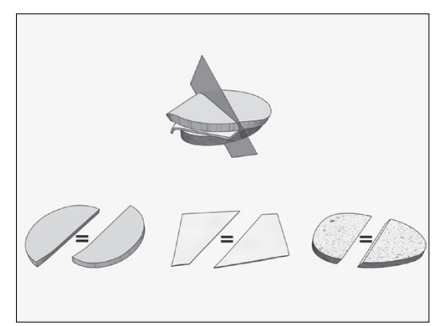

The sandwich problem (the Bolzano-Cauchy theorem).

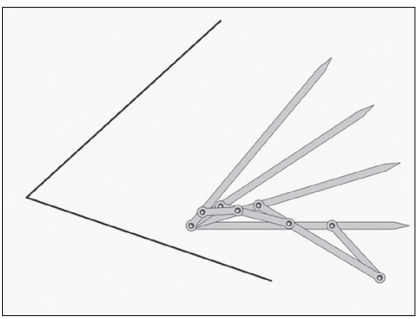

Angle trisection (the theory of linkages). 


\section{"Mechanisms by Tchebyshev"}

An extensive and important topic, designed as a separate subproject, is "Mechanisms by Tchebyshev" (http:// tcheb.ru). All 33 planar linkage mechanisms devised by the great Russian mathematician P.L. Tchebyshev (or Chebyshev in modern writing), as well as various other mechanisms based on these, are demonstrated, explained and modelled using computer graphics. These devices include the world's first walking machine (called a "plantigrade" machine by Tchebyshev), a "sorting machine", a "wheelchair" and a "paddling" mechanism.

Some of these devices and mechanisms are stored in the Polytechnical Museum of Moscow, the Museum of History of Saint Petersburg University, the Museum of Arts and Metiers of Paris and the Science Museum of London. By agreement with these museums, the Laboratory has created computer realisations of these mechanisms based on precise measurements of the original models. Preserving the size of the models, as well as other details of the mechanisms, makes it possible to manufacture (in principle) exact copies of Tchebyshev devices. Mechanisms that appeared to be lost have been reconstructed according to archive documents.

The movies of the project demonstrate both how the mechanisms operate and where they originate from (i.e. their mathematical background). In particular, the idea of an approximation of a desired curve (a line segment, an arc of a circle, a full circle and so on) by a linkage curve played an important role in Tchebyshev's discoveries. He started from a problem, posed by James Watt, of how to construct a linkage between the circular motion and the straight one. Tchebyshev, however, failed to find a precise solution of this problem and then started to develop approximation theory. It is quite remarkable that this technical part of Tchebyshev's activity resulted in the discovery of the celebrated Tchebyshev polynomials, thus initiating a completely new chapter in mathematics! We also hope that the Tchebyshev mechanisms project of our Lab will set a new standard of scientific internetmuseums.

We are happy to have a cupboard in our Lab that used to belong to Tchebyshev, decorated with Tchebyshev's initials and his family's coat of arms. We consider it a symbolic bridge between the era of Tchebyshev and the present time. However, it has not only symbolic meaning but also a quite realistic allocation. In his time, Tchebyshev used it to keep his mechanisms inside. Now, next to the cupboard, we exhibit some of the models described below.

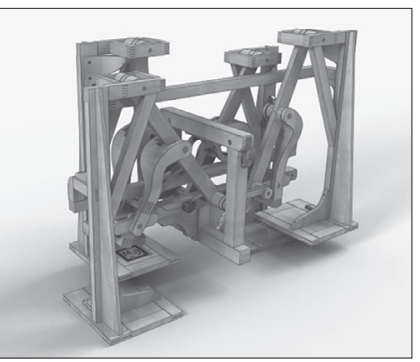

Plantigrade machine.

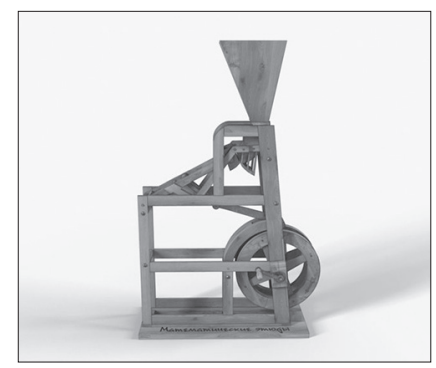

Sorting mechanism.

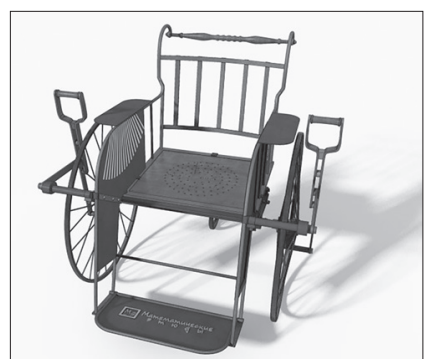

Wheelchair.

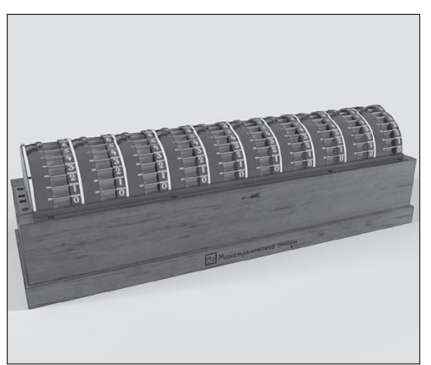

Arithmometer, first model.

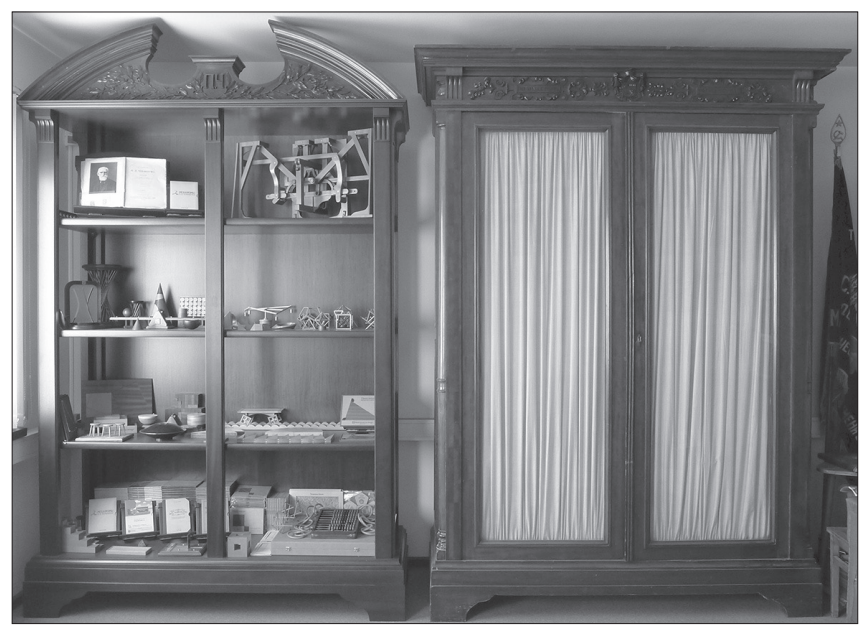

Cupboard of P.L. Tchebyshev.

\section{Models}

In various scientific museums around the world, one can see models demonstrating numerous physical, chemical and biological phenomena directly and in a natural way. However, it is much more difficult to present mathematical models in the same way due to the high level of abstraction of mathematics compared to the other natural sciences.

This makes good mathematical models very significant: they demonstrate the essence of mathematical concepts or statements and they show viewers the hidden mathematical meaning via a series of impressions and sensations, both visual and memorable.

With today's low level of general mathematical education in schools, a visual model and its accessible explanation may help viewers open the gates to the fascinating world of genuine mathematics.

In our collection, we have gathered together some remarkable models made by a real master: Alexander Leshchynskyi. We use some of them in our on-site lectures. Certainly, manipulation with models is a favourite occupation of children and their parents visiting the Laboratory.

Not surprisingly, these models also attract professional mathematicians to the Lab. In particular, during the meeting of the Executive Committee of the European Mathematical Society held in Moscow, all the members of Executive Committee enjoyed the visit to the Lab.

However, since the Laboratory can only accept a restricted number of guests, we have organised the Models Section on the website of "Mathematical Etudes" (http:// etudes.ru/ru/models). In this section, we are also going 
to create a complete electronic catalogue of mathematical models, i.e. bring together all available knowledge of such models including our own achievements. Each model should be illustrated by photos, instructions of how to construct these models and animated representations made using 3D computer graphics. Such animations are already themselves a good teaching tool.

Naturally, our virtual collection should go beyond pure entertainment. Its aim is to help the visitor learn something new from mathematics, to explain mathematically what they see or read. The superficial comments are often the weakest point of modern interactive museums. We hope that the development of a public directory of mathematical models will contribute to the appearance of visual aids in mathematical classrooms and interactive museums.

\section{AppStore Apps}

Mobile devices have become our everyday companions. This opens up new possibilities in mathematics for those who are still not involved, those for whom the traditional ways of teaching mathematics turned out to be ineffective. By developing applications for mobile devices, each user can become the owner of a permanent "mathematical sputnik" - an interlocutor and mentor. We will give several examples of the Laboratory's outcomes in this field.

In Russia, there is a classical book 1001 Problems for Oral Calculations that is well known among mathematicians and mathematics educators. This collection of fascinating arithmetical problems was composed by the outstanding Russian teacher Sergei A. Rachinskii in the late 19th century. Rachinskii was a professor at Moscow University but left it to teach children in a rural school. One of Rachnskii's former students, the Russian artist N.P. Bogdanov-Belsky, painted a rural classroom scene (this painting can now be found in the Moscow Tretyakov Gallery). It shows Rachinskii teaching the peasant students oral arithmetic. A problem is written on the blackboard: $\left(10^{2}+11^{2}+12^{2}+13^{2}+14^{2}\right) / 365$. The peasant children are meant to compute the resulting quantity without pencil, paper or chalk.

The Laboratory has digitised "1001 Problems" and the interactive app for iPhone and iPad has been downloaded by more than 2,000,000 users of ru-Appstore. This is a significant number for the online store, which is on a much smaller scale than its American analogue. Such a great number of paper copies would obviously be impossible in Russia - or indeed anywhere today.

There are several other ME applications (the programming was done by Anton Fonarev and Mark Pervovskiy) available in all regions of the appstore. The Cryptarithms App is a set of mathematical puzzles containing numbers in which digits are substituted by characters. The problem is to replace the characters with digits in order to obtain the correct answer. This is a rather popular entertainment in mathematical circles. The Four Colours app concerns the famous four colour theorem: here one can try to colour various given maps of different countries using only four colours. The Pythagoras App and Pythagoras HD App are puzzles where one tries to prove the Pythagoras theorem.

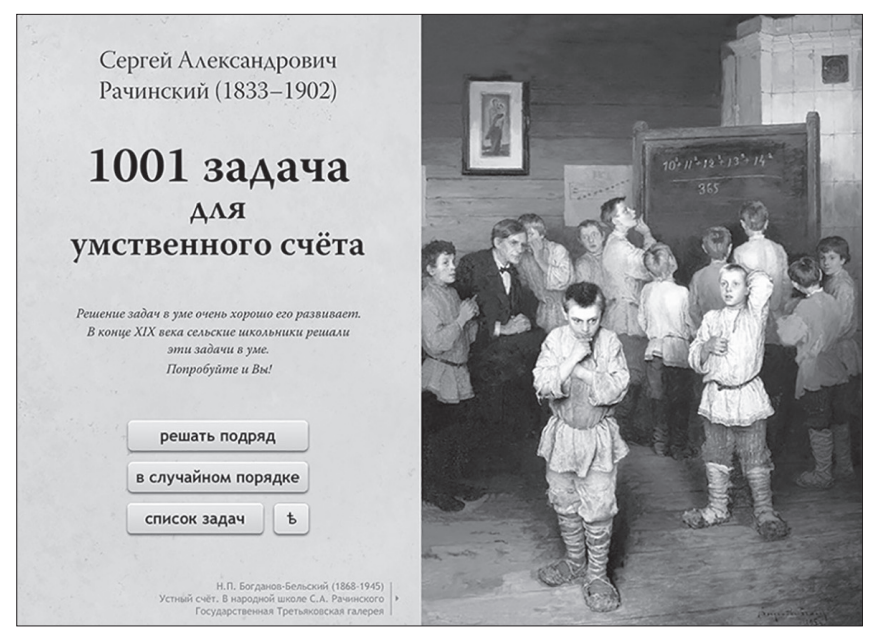

1001 Problems for Oral Calculations.
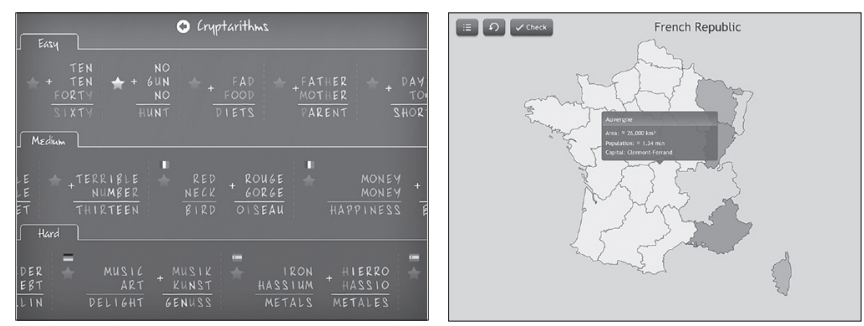

Cryptarithms.

Four Colours.

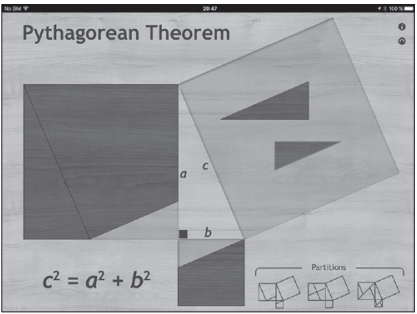

Pythagoras HD.

\section{The Classroom and Beyond}

All the movies are available for free on the "Mathematical Etudes" website. Some of them are translated into Italian, French and English. Others are still waiting to be translated (though one can try to use the automatic translator). The site is visited by more than 15,000 users daily. It is also the entry point to the other Lab projects described above. Different parts of our projects have been demonstrated in more than 700 popular science lectures for school pupils, teachers and university students in different regions of Russia. A standard talk lasts two hours. However, the attending schoolchildren very often ask for an additional class lesson to accompany this lecture. (We all know that maths lessons in school are usually considered the most hard and least understandable!) The Laboratory quite regularly conducts maths lectures on Russian TV based on the Lab products.

Mathematics school teachers in Russia widely use materials of our projects in their classes, as well as in their additional class lessons, in maths circles, at popularscience conferences for school pupils and so on.

Though our Lab projects are mainly directed at school pupils, teachers, university students and educators, our 
experience has shown that even professional mathematicians and other scientists express their interest in the content of projects made in the Laboratory of Popularisation and Promotion of Mathematics of the Steklov Mathematical Institute. We are trying our best to support communication with all visitors to the "Mathematical Etudes" site. We hope that no visitor to this site will be disappointed.

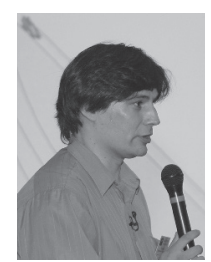

Nikolai Andreev [andreev@etudes.ru] heads the Laboratory of Popularisation and Promotion of Mathematics at the Steklov Mathematical Institute of the Russian Academy of Sciences (RAS) in Moscow. He received his degree in mathematics at the Mechanics and Mathematics Department of the Lomonosov Moscow State University and has worked at the Steklov Institute since 2000. His interests involve approximation theory, coding theory, mathematics popularisation and teaching.

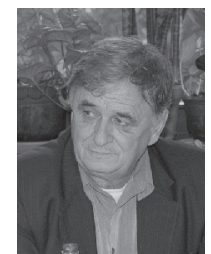

Nikolai Dolbilin [dolbilin@mi.ras.ru] received his PhD in mathematics under B. N. Delaunay from the Steklov Mathematical Institute in Moscow and has worked there since 1969. He is also a professor of mathematics at Moscow State University. His in- terests involve geometry (tilings and Delaunay sets, crystallographic groups, quasicrystalline structures, the theory of polytopes, including parallelohedra), the Ising model, mathematics popularisation and teaching.

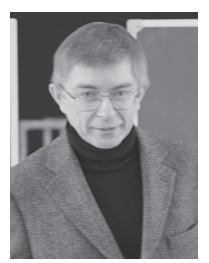

Sergei Konovalov [serk@mi.ras.ru] is a senior scientific researcher at the Laboratory of the Steklov Mathematical Institute and an associate professor in higher mathematics at the Moscow Institute of Physics and Technology. He is a member of the editorial board of "Kvant", the popular-science journal for scholars and students. His scientific interests include function theory, Lie group analysis of differential equations and the history of mathematics. His hobbies include chess and tennis.

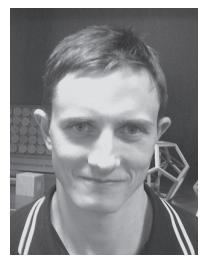

Nikita Panyunin [panyunin@mi.ras.ru] received his degree in mathematics at the Mechanics and Mathematics Department of the Lomonosov Moscow State University. From 2006 to 2010, he worked at the Scientific Research Institute for System Studies of the RAS. Since then, he has worked at the Laboratory of the Steklov Mathematical Institute. In his spare time, he enjoys skiing, running, backpacking trips and playing piano. 